\title{
Contributions to the knowledge of the Lepidoptera of Vlăsia Plain (Muntenia, Romania)
}

\author{
Valeriu Albu \& Sebastian Albu
}

\begin{abstract}
Summary: This study presents the results of an investigation carried out between 1966 and 2013, aiming to record the lepidoptera fauna of the Vlăsia Plain, a region centered on Bucureşti, Romania, and part of the more extensive Valahia Plain. 1251 lepidoptera representing 458 species were collected from forests, steppes, marshy areas and hilly zones situated between Bucureşti and the border of the Carpathian foothills. From these collections, 33 previously unrecorded species were added to the Valahia Plain checklist. Isolated populations of several species previously considered extinct from the area or with a doubtful occurrence, were re-discovered and re-confirmed in wooded habitats near Bucureşti (Lopinga achine and Ancylis upupana), on the hills at Târgovişte (Zerynthia polyxena) and in forest clearings around the city of Piteşti (Brintesia circe).
\end{abstract}

Key words: Lepidoptera biodiversity, European butterflies and moths, Balkan invertebrate fauna, lepidoptera of Romania

\section{Introduction}

The study of the lepidoptera fauna of the Vlăsia Plain (part of larger Muntenia) began in the late 19th century, mainly with the recording of the butterflies and macromoths collected in and around București, the country's capital city (CARADJA 1895, FleCK 1900). Gradually, interest shifted to also include micromoth populations in studies by CARADJA (1901), Popescu (1938), and Popescu-Gorj (1964). These laid the groundwork for the current knowledge of the lepidoptera fauna of București and its surroundings. Unfortunately, this activity came to a halt in the mid1960 s and was not resumed until the late 1990s, with the work of SzÉKeLY (1998, 2002, and 2011). The present study, which primarily documents species collected from 1979 to 1982, attempts to fill this 30year gap in the monitoring of lepidoptera populations of Bucureşti and its surroundings.

\section{Material and methods}

The lepidoptera surveying activities reported in this paper extend over a period of 47 years, from 1966 to 2013. During this time, we investigated 19 sites in four counties situated on the Vlăsia Plain (Ilfov, Dâmbovița, Argeș, and Giurgiu). Table 1 lists these sites and their altitudes along with the number of specimens and species recorded at each of them. Collection sites included electrically illuminated structures, forests, and village gardens in Ilfov, illuminated structures and pastures in Dâmboviţa, wooded areas and clearings in Argeş, and a marshy area in Giurgiu.

We sampled for lepidoptera during the entire flight season, from March to November. Specimens were collected by sweeping vegetation with a standard insect net. Larval specimens were reared to adulthood either on the plants on which they were found or on alternative food sources, e.g. dandelion (Taraxacum officinalis), plantain (Plantgo major), or willow leaves (Salix spp.). Pupae found on rare occasions were retained until eclosion. Sampling events occurred throughout the year, temperature and precipitation permitting.

We followed the species arrangement proposed in „The Lepidoptera of Europe- A Distributional Checklist" (KARSHOLT and RAZOwSKI 1996), with the amendments contained in „Revised higher classification of the Noctuoidea" (LAFONTAINE and FIBIGER 2006) and in Fauna Europaea Web Service v.2.6 (Karsholt and Nieukerken 2013). The "Catalogue of the Lepidoptera of Rumania" (RÁKosY et al. 2003) was used as a benchmark for the species known to occur in our study area.

\section{Results}

We recorded 458 lepidoptera species out of a total of 1251 individuals collected during the entire time span of the study. Of these, 412 species $(89.9 \%)$ and 1156 specimens $(92.5 \%)$ were obtained in the four- 
Table 1. Sampling sites by county with their respective species and specimen numbers, arranged by increasing altitudes. Many species were caught in two or more counties, which accounts for the higher county species sum (584) than the total species collected (458).

\begin{tabular}{ccccc}
\hline County & Site & Altitude & Number of species & Number of specimens \\
\hline Giurgiu & Călugăreni & $58 \mathrm{~m}$ & $85(14.6 \%)$ & $140(11.2 \%)$ \\
\hline Cernica & $60 \mathrm{~m}$ & & \\
Pădurea Pasărea & $70 \mathrm{~m}$ & & \\
Ilfov & Fundeni & $80 \mathrm{~m}$ & & \\
& Chitila & $90 \mathrm{~m}$ & & \\
& Pădurea Andronache & $90 \mathrm{~m}(72.3 \%)$ & \\
& Pădurea Mogoşoaia & $95 \mathrm{~m}$ & \multirow{2}{*}{$362(62.0 \%)$} & \\
& Otopeni & $95 \mathrm{~m}$ & & \\
& Ramificaţia Buciumeni & $95 \mathrm{~m}$ & & \\
& Ciorogârla & $97 \mathrm{~m}$ & & \\
& Săbăreni & $100 \mathrm{~m}$ & & \\
& Pădurea Râioasa & $100 \mathrm{~m}$ & & \\
& Băneşti & $182 \mathrm{~m}$ & & \\
Dâmbovița & Cazaci & $210 \mathrm{~m}$ & & \\
& Târgovişte & $280 \mathrm{~m}$ & $103(17.6 \%)$ & \\
& Cozia & $288 \mathrm{~m}$ & & 1251 \\
\hline \multirow{2}{*}{ Argeș } & Găieşti & $347 \mathrm{~m}$ & & \\
\hline Total & Drăganu & $390 \mathrm{~m}$ & \multirow{2}{*}{$34(5.8 \%)$} & \\
\hline
\end{tabular}

Table 2. Number of species collected during this study, with corresponding suprafamily designations.

\begin{tabular}{ccc}
\hline & Suprafamily & Number of species \\
\hline 1 & Noctuoidea & 134 \\
2 & Geometroidea & 96 \\
3 & Papilionoidea & 66 \\
4 & Pyraloidea & 49 \\
5 & Tortricoidea & 26 \\
6 & Gelechioidea & 16 \\
7 & Adeloidea & 11 \\
8 & Bombycoidea & 9 \\
9 & Tineoidea & 7 \\
10 & Gracillarioidea & 7 \\
11 & Yponomeutoidea & 6 \\
12 & Cossoidea & 6 \\
13 & Zygaenoidea & 6 \\
14 & Drepanoidea & 6 \\
15 & Pterophoroidea & 4 \\
16 & Lasiocampoidea & 4 \\
17 & Eriocranioidea & 1 \\
18 & Hepialoidea & 1 \\
19 & Tischeroidea & 1 \\
20 & Choreutoidea & 1 \\
21 & Thyridoidea & 1 \\
\hline Total & & 458 \\
\hline 6 & &
\end{tabular}

year interval between 1979 to 1982 . We recorded the most species from Ilfov (362), followed by Dâmboviţa (103), Giurgiu (85), and Argeș (34), as detailed in Table 1. Many species were collected in two or more counties. These species represent 21 suprafamilies, as shown in Table 2. The Noctuoidea and Geometroidea had the highest numbers of species in our sample with 134 and 96, followed by Papilionoidea and Pyraloidea with 66 and 49, respectively. At the bottom were Eriocranioidea, Hepialoidea, Tischeroidea, Choreutoidea, and Thyridoidea with one species for each. We recorded lepidoptera activity from March to December, with the most species collected during May and June (Fig. 1). This graph shows a steep increase in species abundance from March to June, followed by a decrease in July, another small peak in August, and a gradual decrease in abundance from September to December. The only month devoid of any records was January. In February 1983 we had a Saturnia pavonia (LinNaEus, 1758) pupa hatch indoors and in December 1980 and 1981 we collected single specimens of Agonopterix alstromeriana (CleRCK, 1759) and Erannis defoliaria (CLERCK, 1759), respectively. 


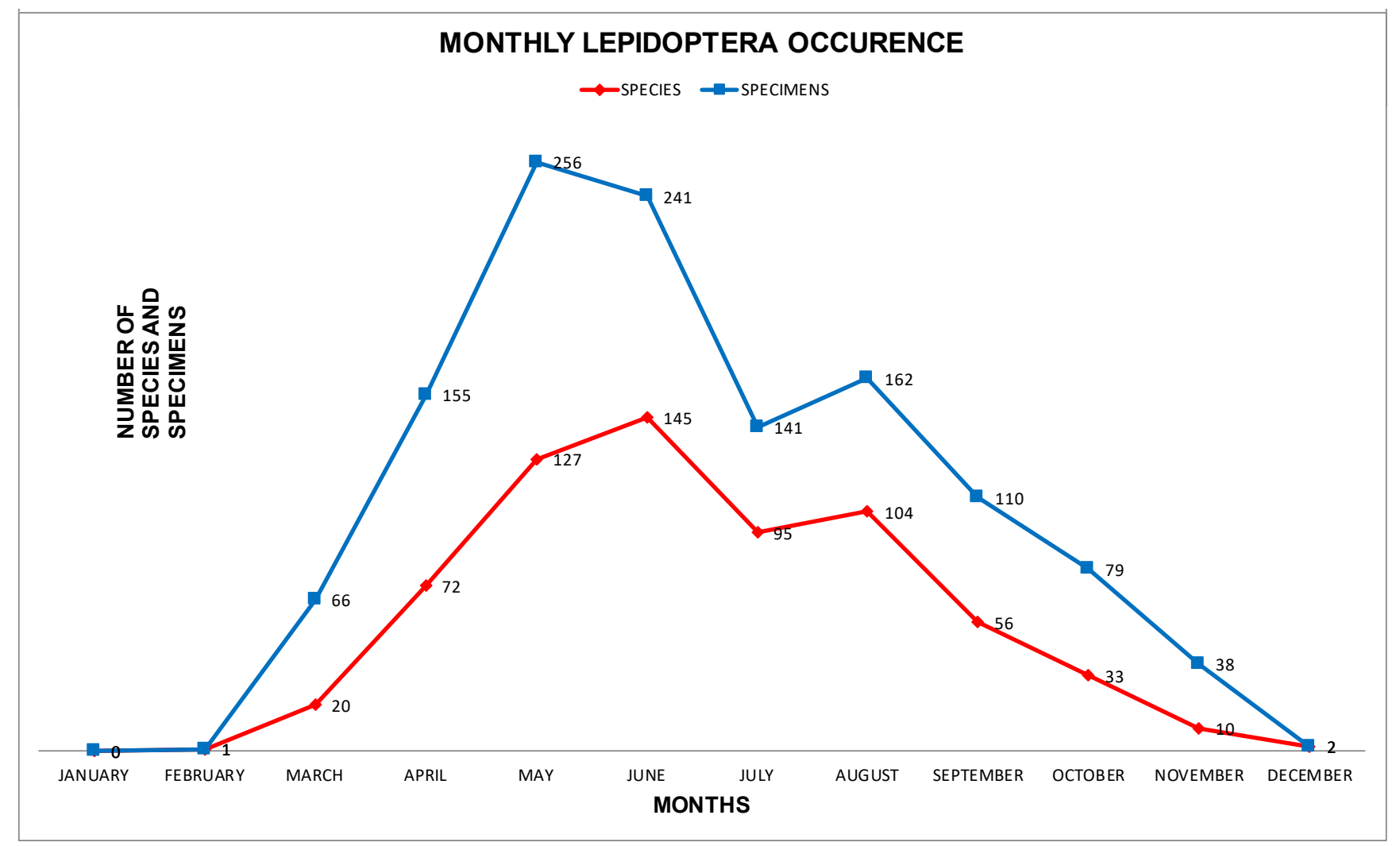

Fig. 1. Line graph depicting monthly differences in numbers of lepidoptera species and specimens collected during this study. Many species were caught in more than one month, resulting in a higher monthly sum (665) than the total species number for the study (458).

\section{Discussion}

During this study, we recorded 458 lepidoptera species from the Vlăsia plain. Of these, 421 were species known to regularly occur in the area, while 33 were previously unrecorded from here, as well as from Muntenia (Table 3). Four more species were encountered in places from where they were considered extirpated. We rediscovered Ancylis upupana (TreitschKe, 1835) in Andronache forest in 1982. The last record of this species from Muntenia was prior to 1900 (RÁkosy et al. 2003). We encountered several populations of Lopinga achine (SCOPOLI, 1763) in Mogoşoaia and Andronache forests near Bucureşti in 1981 and 1982 and of Zerynthia polyxena (DENIS and SCHIFFERMÜLLER, 1775) in the foothills at Mânăstirea Dealu near Târgovişte in 1982. These two species had not been seen from these areas since the beginning of the 20th century and were considered extinct from here (Popescu-GoRj 1964, RÁKosy 2013). There are a few even more recent records of $L$. achine from 1997 to 2004 reported from this area by SzÉKELY (2011) and at least one more record of $Z$. polyxena from Târgoviște in 1987 (personal observation). We documented the occurrence of Brintesia circe (FABRICIUS, 1775) in Argeş, a species which had a questionable presence in the area (RÁkosy 2013). This suggests the presence and persistence of these species in Muntenia, most likely as localized and isolated populations.

Our data suggest that the most and least annual lepidoptera flight activity occurred in June (145 species) and January (0 species), respectively. The monthly species abundance curve (Fig. 1) shows an increase in lepidoptera flight activity from March to June and a decrease from September to December, similar to that observed in the study of the lepidoptera of Bucureşti (Albu and Albu 2016). We observed sporadic activity in December, as shown by the records of $A$. alstromeriana and E. defoliaria in (1980 and 1981, respectively). The former species overwinters as adult, while the latter flies in the late fall, making a December detection not unusual, given the occasional occurrence of warm days during this month. The February record of a $S$. pavonia adult hatching indoors is also consistent with personal observations of wild individuals of this species flying in March and April.

RÁKosy et al. (2003) compiled a list of 1916 lepidoptera species from the existing literature and museum collections representing the fauna of the entire Muntenia region, thus also covering our study area. Adding the 458 species from this study to the 78 nonduplicated species that we encountered by surveying an urban park in Bucureşti during approximately the same period (Albu and Albu 2016), we arrive at 536 lepidoptera species recorded from the Vlăsia plain in the latter part of the 20th century. This represents $28 \%$ of the total of 1916 species found by RÁKOSY (2003). Some species, including Noctua haywardi (TAMs, 1926) were recorded just recently in this area 


\begin{tabular}{|c|c|c|c|c|c|c|c|}
\hline Suprafamily & K \& R Number & Taxon & County & Locality & Year & Month & Day \\
\hline Adeloidea & 366 & Adela cuprella & Dâmboviţa & Târgovişte, Mânăstirea Dealu & 1982 & May & 20 \\
\hline Tischerioidea & 442 & Tischeria decidua & Giurgiu & Călugăreni & 1982 & May & 16 \\
\hline \multirow{3}{*}{ Tineoidea } & 613 & Triaxomera fulvimitrella & Argeş & Cozia & 2007 & May & 26 \\
\hline & 461 & Myrmecozela ochraceella & Ilfov & Pădurea Râioasa & 1982 & May & 21 \\
\hline & 748 & Diplodoma laichartingella & Dâmboviţa & Târgovişte, Mânăstirea Dealu & 1982 & May & 4 \\
\hline \multirow{4}{*}{ Gracillarioidea } & 1090 & Bucculatrix rhamniella & Dâmboviţa & Târgovişte, Mânăstirea Dealu & 1981 & October & 7 \\
\hline & 1110 & Caloptilia alchimiella & Ilfov & Pădurea Mogoşoaia & 1981 & May & 15 \\
\hline & 1131 & Caloptilia stigmatella & Ilfov & Bucureşti & 1980 & June & 20 \\
\hline & 1297 & Phyllonorycter roboris & Ilfov & Pădurea Mogoşoaia & 1980 & April & 29 \\
\hline Yponomeutoidea & 1408 & Paraswammerdamia nebulella & Ilfov & Pădurea Mogoşoaia & 1981 & April & 14 \\
\hline \multirow{7}{*}{ Gelechioidea } & 2131 & Scythris tributella & Argeş & Drăganu & 2007 & May & 25 \\
\hline & 2234 & Dasystoma salicella & Ilfov & Pădurea Râioasa & 1982 & April & 7 \\
\hline & 2298 & Crassa tinctella & Ilfov & Pădurea Râioasa & 1982 & May & 21 \\
\hline & 2911 & Hypatopa inunctella & Ilfov & Ramificaţia Buciumeni, Chitila & 1981 & June & 5 \\
\hline & 3154 & Limnaecia phragmitella & Ilfov & Otopeni & 2002 & June & 22 \\
\hline & 3280 & Metzneria metzneriella & Ilfov & Ramificaţia Buciumeni, Chitila & 1982 & September & 7 \\
\hline & 3304 & Psammathocrita osseella & Ilfov & Pădurea Pasărea & 1982 & May & 21 \\
\hline \multirow{5}{*}{ Tortricoidea } & 4409 & Acleris literana & Ilfov & Pădurea Mogoşoaia & 1982 & March & 26 \\
\hline & 4659 & Bactra robustana & Argeş & Drăganu & 2007 & May & 25 \\
\hline & 4963 & Eucosma conterminana & Ilfov & Otopeni & 2002 & June & 22 \\
\hline & 5019 & Notocelia cynosbatella & Ilfov & Pădurea Pasărea & 1981 & May & 24 \\
\hline & 5025 & Notocelia rosaecolana & Argeş & Cozia & 2007 & May & 26 \\
\hline \multirow{2}{*}{ Pterophoridea } & 5434 & Cnaemidophorus rhododactyla & Ilfov & Pădurea Pasărea & 1976 & July & 4 \\
\hline & 5445 & Oxyptilus distans & Ilfov & Chitila & 1979 & September & 22 \\
\hline Pyraloidea & 5724 & Sciota rhenella & Ilfov & Ramificaţia Buciumeni, Chitila & 1981 & June & 9 \\
\hline \multirow{2}{*}{ Drepanoidea } & 7494 & Polyploca ridens & Ilfov & Ramificaţia Buciumeni, Chitila & 1981 & April & 7 \\
\hline & 7496 & Asphalia ruficollis & Ilfov & Ramificaţia Buciumeni, Chitila & 1981 & March & 24 \\
\hline \multirow{6}{*}{ Geometroidea } & 7681 & Lycia pomonaria & Ilfov & Ramificaţia Buciumeni, Chitila & 1981 & March & 24 \\
\hline & 7693 & Agriopis leucophaearia & Ilfov & Pădurea Mogoşoaia & 1981 & March & 10 \\
\hline & 7829 & Lomographa temerata & Ilfov & Pădurea Mogoşoaia & 1981 & May & 18 \\
\hline & 8038 & Scopula corrivalaria & Ilfov & Ramificaţia Buciumeni, Chitila & 1981 & August & 1 \\
\hline & 8401 & Horisme corticata & Dâmboviţa & Târgovişte, Mânăstirea Dealu & 1982 & May & 20 \\
\hline & 8658 & Asthena anseraria & Dâmboviţa & Târgovişte, Mânăstirea Dealu & 1982 & June & 17 \\
\hline
\end{tabular}


(SzÉKelY 2002), while others such as Saturnia spini (DENIS and SCHIFFERMÜLLER, 1775) disappeared from the region in the 20th century (RÁKosy et al. 2003). The disappearance of species should be considered with caution, however. After many years of lacking records, our collections of L. achine, Z. polyxena, and A. upupana show how increased sampling can reveal the presence of species considered extinct from an area.

According to the Romanian lepidoptera catalogue (RÁKosy et al. 2003), there are 2453 „Microlepidoptera” (pre-Lasiocampoid) and 1507 „Macrolepidoptera” (post-Lasiocampoid) species sensu Karsholt and RazowsKi (1996) known to occur in Romania. Of these, 874 „Microlepidoptera” and 1042 „Macrolepidoptera" species $(35 \%$ and $69 \%$, respectively) are known from Muntenia. For comparison, there are 1708 „Microlepidoptera” and 1277 „Macrolepidoptera” species $(69 \%$ and $84 \%$, respectively) listed from Transylvania and 1414 „Microlepidoptera" and 1192 „Macrolepidoptera” species (57\% and 79\% respectively) from Moldova, both regions comparable in size and relief to Muntenia. This makes the „Microlepidoptera” fauna of Muntenia one of the least known of Romania. Even allowing for the extensive losses in various habitats to urbanization and agriculture, we posit a higher lepidoptera species richness (especially „Microlepidoptera”) for Muntenia than what the records suggest. This underscores the importance of carrying out additional surveys, especially investigating the „Microlepidoptera” fauna, to gain a better understanding of its composition and of the population and species shifts that are occurring in this area.

\section{Acknowledgements}

We would like to express our gratitude to Jean-François LANDRY for his help in identifying the Coleophoridae and Scythrididae and to Cees GIELIS for clarifying the identity of the Pterophoridae species encountered during this study. We extend our thanks to Alexander ALBU for reviewing this manuscript.

\section{Bibliography}

Albu V. and Albu S. (2016) Contributions to the knowledge of the Lepidoptera species diversity in an urban park setting of Bucharest, Romania, with considerations on the species dynamics in the city over the last century. Entomologica Romanica 20: 69-78. Published online.

Caradja A. (1895) Die Großschmetterlinge des Königreiches Rumänien. [The Macrolepidoptera of the Kingdom of Romania]. Deutsche Entomologische Zeitschrift Iris, Dresden, 8: 1-102 (in German).

FLEC E. (1900) Die Makrolepidopteren Rumäniens. [The Macrolepidoptera of Romania]. Buletinul Societăţii de Sciinţe din Bucuresci-România 9(1): 1-200 (in German).

KARSHOLT O. and RAZOWSKI J. (eds). (1996) The Lepidoptera of Europe - A distributional Checklist. Apollo Books, Stenstrup. Denmark.

Karsholt O. and NieUKerKen E.J. van. (2013) Lepidoptera, Moths. Fauna Europaea version 2.6, http://fauna-eu. org.

LAFOnTAINE J.D. and Fibiger M. (2006) Revised higher classification of the Noctuoidea (Lepidoptera). Canadian Entomologist 138: 610-635.

PopescuA.A. (1938) Noi contribuţii la fauna Lepidopterelor din Bucureşti şi împrejurimi [New contributions to the lepidoptera fauna of Bucharest and its surroundings]. Academia Română, Memoriile Secţiunii Ştiinţifice, seria 3-4(3): 1-24, Bucureşti (in Romanian).

Popescu-GorJ A. (1964) Catalogue de la collection de lépidoptères „Prof. A. Ostrogovich” du Muséum d'Histoire Naturelle „Grigore Antipa”, Bucarest. [The cataloue of the lepidoptera collection „Prof. A. Ostrogovich” of the „Grigore Antipa” Natural History Museum of Bucharest]. Editura Muzeului „Grigore Antipa”, Bucureşti.

Rákosy L., GoiA M. and Kovács Z. (2003) Catalogul Lepidopterelor României [Catalogue of the Lepidoptera of Romania]. Societatea Lepidopterologică Română, Cluj-Napoca (bilingual Romanian and German).

RÁKosy L. (2013) Fluturii diurni din România. Cunoaștere, protecție, conservare. [The Butterflies of Romania]. Editura Mega, Cluj-Napoca.

SzÉKElY L., HANESCHLÄGER P. and GöRBE R. (1998) Contribuţii la studiul faunei de lepidoptere din Câmpia Munteniei. I. [Contributions to the study of the Lepidoptera fauna of the Muntenia Plain. I.] Buletin Informativ. Societatea Lepidopterologică Română 9(34): 177-186 (in Romanian).

SzÉKELY L. and STANCIU S.M. (2002) Contribuţii la studiul faunei de lepidoptere din Câmpia Munteniei. II. [Contributions to the study of the Lepidoptera fauna of the Muntenia Plain. II.] Buletin Informativ, Societatea Lepidopterologică Română 12(1-4): 259-269 (in Romanian).

SzÉKELY L. (2011) The Lepidoptera of Bucharest and its surroundings (Romania). Travaux du Muséum National d'Histoire Naturelle ,, Grigore Antipa” 54(2): 461-512.
Valeriu ALBU

23032 Oak Meadow Ln., Friant,

CA 93626

E-mail:valalbu@netptc.net

\author{
Sebastian Albu \\ California Department of Food and \\ Agriculture Plant Health and Pest \\ Prevention Services \\ Plant Pest Diagnostics Lab \\ 3294 Meadowview Road \\ Sacramento, CA 95832-1448 \\ E-mail: sebastian.albu@cdfa.ca.gov
}


Appendix 1. Lepidoptera species of the Vlăsia Plain (Muntenia) and their county distributions. K \& R refers to the KARSHOLT and RAZOWSKI (1996) checklist.

\begin{tabular}{|c|c|c|c|c|c|c|}
\hline \multirow[t]{2}{*}{ Suprafamily } & \multirow{2}{*}{$\begin{array}{c}\text { K\& R } \\
\text { Number }\end{array}$} & \multirow[t]{2}{*}{ Taxon } & \multicolumn{4}{|c|}{ County } \\
\hline & & & Ilfov & Dâmbovița & Giurgiu & Argeș \\
\hline Eriocranioidea & 48 & Dyseriocrania subpurpurella & 1 & & & \\
\hline Hepialoidea & 63 & Triodia sylvina & 1 & & & \\
\hline \multirow{11}{*}{ Adeloidea } & 338 & Nemophora degeerella & 1 & & & \\
\hline & 352 & Nemophora fasciella & 1 & & & \\
\hline & 365 & Adela reaumurella & 1 & & & \\
\hline & 366 & Adela cuprella & & 1 & & \\
\hline & 377 & Cauchas fibulella & 1 & 1 & & \\
\hline & 378 & Cauchas leucocerella & 1 & 1 & & \\
\hline & 382 & Cauchas rufimitrella & & 1 & & \\
\hline & 385 & Nematopogon pilella & 1 & & & \\
\hline & 389 & Nematopogon adansoniella & & 1 & & \\
\hline & 391 & Nematopogon swammerdamella & 1 & 1 & & \\
\hline & 424 & Incurvaria masculella & & 1 & & \\
\hline Tischerioidea & 442 & Tischeria decidua & & & 1 & \\
\hline \multirow{7}{*}{ Tineoidea } & 747 & Diplodoma laichartingella & & 1 & & \\
\hline & 415 & Psychidea nudella & 1 & & & \\
\hline & 609 & Morophaga choragella & 1 & & & \\
\hline & 613 & Triaxomera fulvimitrella & & & & 1 \\
\hline & 669 & Tineola bisselliella & 1 & & & \\
\hline & 689 & Niditinea fuscella & 1 & & & \\
\hline & 724 & Euplocamus anthracinalis & 1 & & & \\
\hline \multirow{7}{*}{ Gracillarioidea } & 1070 & Bucculatrix frangutella & & 1 & & \\
\hline & 1090 & Bucculatrix rhamniella & & 1 & & \\
\hline & 1110 & Caloptilia alchimiella & 1 & & 1 & \\
\hline & 1131 & Caloptilia stigmatella & 1 & & & \\
\hline & 1135 & Gracillaria syringella & 1 & & & \\
\hline & 1172 & Callisto denticulella & 1 & & & \\
\hline & 1297 & Phyllonorycter roboris & 1 & & & \\
\hline \multirow{6}{*}{ Yponomeutoidea } & 1348 & Yponomeuta padella & 1 & & & \\
\hline & 1408 & Paraswammerdamia nebulella & 1 & & & \\
\hline & 1458 & Argyresthia curvella & 1 & & & \\
\hline & 1525 & Plutella xylostella & 1 & & & \\
\hline & 1576 & Orthotelia sparganella & 1 & & & \\
\hline & 1499 & Ypsolopha chazariella & 1 & & & \\
\hline \multirow{16}{*}{ Gelechioidea } & 2911 & Hypatopa inunctella & 1 & & & \\
\hline & 2298 & Crassa tinctella & 1 & & & \\
\hline & 2322 & Alabonia staintoniella & 1 & & 1 & 1 \\
\hline & 2231 & Diurnea fagella & 1 & 1 & & \\
\hline & 2234 & Dasystoma salicella & 1 & & & \\
\hline & 1674 & Luquetia lobella & & 1 & & \\
\hline & 1730 & Agonopterix alstromeriana & 1 & 1 & & \\
\hline & 1644 & Ethmia quadrillella & 1 & & & \\
\hline & 2892 & Mompha epilobiella & 1 & & & \\
\hline & 2131 & Scythris tributella & & & & 1 \\
\hline & 2169 & Scythris limbella & 1 & & & \\
\hline & 3154 & Limnaecia phragmitella & 1 & & & \\
\hline & 3252 & Atramaea lonchoptera & 1 & & & \\
\hline & 3280 & Metzneria metzneriella & 1 & & & \\
\hline & 3304 & Psammathocrita osseella & 1 & & & \\
\hline & 3400 & Recurvaria leucatella & 1 & & & \\
\hline
\end{tabular}




\begin{tabular}{|c|c|c|c|c|c|c|}
\hline \multirow[t]{2}{*}{ Suprafamily } & \multirow{2}{*}{$\begin{array}{c}\text { K \& R } \\
\text { Number }\end{array}$} & \multirow[t]{2}{*}{ Taxon } & \multicolumn{4}{|c|}{ County } \\
\hline & & & Ilfov & Dâmbovița & Giurgiu & Argeș \\
\hline \multirow{4}{*}{ Pterophoroidea } & 5434 & Cnaemidophorus rhododactyla & 1 & & & \\
\hline & 5445 & Oxyptilus distans & 1 & & 1 & \\
\hline & 5485 & Pterophorus pentadactyla & 1 & & & \\
\hline & 5552 & Emmelina monodactyla & 1 & & 1 & 1 \\
\hline \multirow[t]{13}{*}{ Choreutoidea } & 5269 & Anthophila fabriciana & 1 & & & 1 \\
\hline & 4255 & Phalonidia manniana & 1 & & & \\
\hline & 4268 & Agapeta hamana & 1 & & & \\
\hline & 4271 & Agapeta zoegana & & & & 1 \\
\hline & 4409 & Acleris literana & 1 & & & \\
\hline & 4471 & Cnephasia incertana & 1 & & & \\
\hline & 4520 & Eulia ministrana & 1 & & & \\
\hline & 4559 & Archips xylosteana & & & 1 & \\
\hline & 4574 & Ptycholoma lecheana & 1 & & & \\
\hline & 4579 & Pandemis cerasana & & 1 & & \\
\hline & 4584 & Syndemis musculana & 1 & & & \\
\hline & 4656 & Bactra furfurana & & & 1 & \\
\hline & 4659 & Bactra robustana & & & & 1 \\
\hline \multirow{16}{*}{ Tortricoidea } & 4713 & Hedya salicella & & 1 & & \\
\hline & 4731 & Celypha lacunana & & & & 1 \\
\hline & 4733 & Celypha rivulana & & & 1 & 1 \\
\hline & 4776 & Olethreutes arcuella & 1 & & 1 & \\
\hline & 4875 & Epinotia tedella & & & & 1 \\
\hline & 4963 & Eucosma conterminana & 1 & & & \\
\hline & 4994 & Epiblema scutulana & 1 & & & \\
\hline & 4998 & Epiblema foenella & 1 & & & \\
\hline & 5019 & Notocelia cynosbatella & 1 & & & \\
\hline & 5025 & Notocelia rosaecolana & & & & 1 \\
\hline & 5063 & Ancylis upupana & 1 & & & \\
\hline & 5073 & Ancylis badiana & & & & 1 \\
\hline & 5076 & Ancylis mitterbacheriana & 1 & & & \\
\hline & 5144 & Cydia pomonella & 1 & & & \\
\hline & 4178 & Phragmataecia castaneae & 1 & & & \\
\hline & 4059 & Synanthedon vespiformis & 1 & & & \\
\hline \multirow{6}{*}{ Cossoidea } & 4064 & Synanthedon tipuliformis & 1 & & & \\
\hline & 4125 & Chamaesphecia annellata & 1 & & & \\
\hline & 4130 & Chamaesphecia bibioniformis & 1 & & & \\
\hline & 4140 & Chamaesphecia empiformis & 1 & & & \\
\hline & 3907 & Apoda limacodes & 1 & & & \\
\hline & 3956 & Adscita statices & & & & 1 \\
\hline \multirow{4}{*}{ Zygaenoidea } & 3974 & Zygaena purpuralis & 1 & & & \\
\hline & 3983 & Zygaena loti & 1 & 1 & 1 & \\
\hline & 3995 & Zygaena ephialtes & 1 & & 1 & \\
\hline & 3998 & Zygaena filipendulae & 1 & & 1 & \\
\hline \multirow[t]{5}{*}{ Thyridoidea } & 5562 & Thyris fenestrella & 1 & 1 & & \\
\hline & 6945 & Zerynthia polyxena & & 1 & & \\
\hline & 6953 & Parnassius mnemosyne & 1 & & 1 & \\
\hline & 6958 & Iphiclides podalirius & 1 & 1 & & \\
\hline & 6960 & Papilio machaon & 1 & & & \\
\hline \multirow{5}{*}{ Papilionoidea } & 6879 & Erynnis tages & 1 & 1 & & \\
\hline & 6882 & Carcharodus alceae & 1 & & & \\
\hline & 6904 & Pyrgus malvae & 1 & 1 & 1 & \\
\hline & 6924 & Thymelicus sylvestris & & 1 & & \\
\hline & 6930 & Ochlodes sylvanus & 1 & & & \\
\hline
\end{tabular}




\begin{tabular}{|c|c|c|c|c|c|c|}
\hline \multirow[t]{2}{*}{ Suprafamily } & \multirow{2}{*}{$\begin{array}{c}\text { K\& R } \\
\text { Number }\end{array}$} & \multirow[t]{2}{*}{ Taxon } & \multicolumn{4}{|c|}{ County } \\
\hline & & & Ilfov & Dâmbovița & Giurgiu & Argeș \\
\hline & 6966 & Leptidea sinapis & & 1 & & \\
\hline & 6973 & Anthocharis cardamines & 1 & & 1 & \\
\hline & 6993 & Aporia crataegi & 1 & & & \\
\hline & 7005 & Pontia edusa & 1 & & 1 & \\
\hline & 7014 & Colias erate & 1 & & 1 & \\
\hline & 7015 & Colias croceus & 1 & & 1 & \\
\hline & 7021 & Colias hyale & 1 & 1 & & 1 \\
\hline & 7022 & Colias alfacariensis & 1 & & & \\
\hline & 7024 & Gonepteyx rhamni & & & 1 & \\
\hline & 7030 & Hamearis lucina & 1 & & & \\
\hline & 7034 & Lycaena phlaeas & 1 & & & \\
\hline & 7036 & Lycaena dispar & 1 & 1 & 1 & \\
\hline & 7043 & Lycaena thersamon & 1 & & & \\
\hline & 7047 & Thecla betulae & 1 & & & \\
\hline & 7058 & Callophrys rubi & & 1 & & \\
\hline & 7062 & Satyrium w-album & & 1 & & \\
\hline & 7063 & Satyrium pruni & 1 & 1 & & \\
\hline & 7065 & Satyrium ilicis & 1 & & & \\
\hline & 7067 & Satyrium acaciae & 1 & 1 & & 1 \\
\hline & 7088 & Cupido minimus & 1 & & & \\
\hline & 7093 & Cupido argiades & & 1 & 1 & \\
\hline & 7095 & Cupido alcetas & & & 1 & \\
\hline & 7097 & Celastrina argiolus & 1 & 1 & 1 & \\
\hline & 7107 & Glaucopsyche alexis & & 1 & & \\
\hline & 7127 & Plebejus argus & 1 & & & \\
\hline & 7128 & Plebejus idas & 1 & 1 & & \\
\hline \multirow[t]{27}{*}{ Papilionoidea } & 7145 & Aricia agestis & 1 & & 1 & \\
\hline & 7152 & Cyaniris semiargus & & 1 & & \\
\hline & 7163 & Polyommatus icarus & 1 & & 1 & \\
\hline & 7172 & Lysandra bellargus & & 1 & & \\
\hline & 7312 & Lasiommata maera & 1 & 1 & & \\
\hline & 7315 & Lopinga achine & 1 & & & \\
\hline & 7325 & Coenonympha arcania & 1 & 1 & & \\
\hline & 7326 & Coenonympha glycerion & & 1 & & \\
\hline & 7344 & Aphantopus hyperantus & & 1 & & \\
\hline & 7350 & Maniola jurtina & 1 & & 1 & \\
\hline & 7427 & Minois dryas & 1 & & 1 & \\
\hline & 7447 & Brintesia circe & & & & 1 \\
\hline & 7202 & Argynnis paphia & 1 & & & 1 \\
\hline & 7210 & Issoria lathonia & & & 1 & \\
\hline & 7213 & Brenthis ino & 1 & & & \\
\hline & 7214 & Brenthis daphne & & 1 & & \\
\hline & 7222 & Boloria selene & 1 & & & \\
\hline & 7228 & Boloria dia & 1 & 1 & & \\
\hline & 7290 & Neptis sappho & & 1 & & \\
\hline & 7297 & Apatura metis & & & 1 & \\
\hline & 7257 & Nymphalis antiopa & & 1 & & \\
\hline & 7258 & Nymphalis polychloros & 1 & & & \\
\hline & 7248 & Aglais io & 1 & & & \\
\hline & 7243 & Vanessa atalanta & & & 1 & \\
\hline & 7245 & Vanessa cardui & 1 & & & \\
\hline & 7252 & Polygonia c-album & 1 & 1 & & \\
\hline & 7255 & Araschnia levana & 1 & & & \\
\hline
\end{tabular}




\begin{tabular}{|c|c|c|c|c|c|c|}
\hline \multirow[t]{2}{*}{ Suprafamily } & \multirow{2}{*}{$\begin{array}{c}\text { K\& R } \\
\text { Number }\end{array}$} & \multirow[t]{2}{*}{ Taxon } & \multicolumn{4}{|c|}{ County } \\
\hline & & & Ilfov & Dâmbovița & Giurgiu & Argeș \\
\hline \multirow{4}{*}{ Papilionoidea } & 7271 & Melitaea phoebe & 1 & & & \\
\hline & 7274 & Melitaea trivia & 1 & & & \\
\hline & 7283 & Melitaea athalia & 1 & 1 & & \\
\hline & 7266 & Euphydryas maturna & 1 & & & \\
\hline \multirow{49}{*}{ Pyraloidea } & 5620 & Synaphe punctalis & & & & 1 \\
\hline & 5627 & Pyralis farinalis & 1 & & & \\
\hline & 5656 & Hypsopygia rubidalis & 1 & & & \\
\hline & 5658 & Hypsopygia glaucinalis & 1 & & & \\
\hline & 5574 & Lamoria zelleri & 1 & & & \\
\hline & 5578 & Lamoria anella & 1 & & & \\
\hline & 5751 & Oncocera semirubella & 1 & & & \\
\hline & 5724 & Sciota rhenella & 1 & & & \\
\hline & 6072 & Homoeosoma sinuella & & & 1 & \\
\hline & 6079 & Homoeosoma nebulella & 1 & & & \\
\hline & 6145 & Ematheudes punctella & 1 & & & 1 \\
\hline & 6162 & Cholius luteolaris & & 1 & & \\
\hline & 6163 & Scoparia manifestella & 1 & & & \\
\hline & 6165 & Scoparia subfusca & & 1 & & \\
\hline & 6172 & Scoparia pyralella & & 1 & 1 & \\
\hline & 6222 & Chilo phragmitella & 1 & & & \\
\hline & 6235 & Calamotropha paludella & 1 & & & \\
\hline & 6207 & Euchromius ocellea & 1 & & & \\
\hline & 6241 & Chrysoteuchia culmella & 1 & & & \\
\hline & 6243 & Crambus pascuella & 1 & & & \\
\hline & 6258 & Agriphila tristella & 1 & & & \\
\hline & 6260 & Agriphila inquinatella & & & 1 & \\
\hline & 6266 & Agriphila selasella & 1 & & & \\
\hline & 6344 & Chrysocrambus linetella & 1 & & & \\
\hline & 6355 & Pediasia luteella & 1 & & & \\
\hline & 6364 & Pediasia contaminella & 1 & & & \\
\hline & 6396 & Scirpophaga praelata & & & 1 & \\
\hline & 6416 & Elophila nymphaeata & 1 & & & \\
\hline & 6423 & Cataclysta lemnata & 1 & & & \\
\hline & 6441 & Aporodes floralis & 1 & & & \\
\hline & 6499 & Evergestis extimalis & 1 & & 1 & \\
\hline & 6507 & Evergestis aenealis & & & 1 & 1 \\
\hline & 6577 & Loxostege sticticalis & 1 & & & \\
\hline & 6588 & Ecpyrrhorrhoe rubiginalis & 1 & & & \\
\hline & 6601 & Pyrausta despicata & 1 & & & \\
\hline & 6604 & Pyrausta aurata & 1 & 1 & 1 & 1 \\
\hline & 6605 & Pyrausta purpuralis & 1 & 1 & 1 & 1 \\
\hline & 6623 & Sitochroa palealis & 1 & & 1 & \\
\hline & 6624 & Sitochroa verticalis & 1 & & & \\
\hline & 6641 & Sclerocona acutellus & 1 & & & \\
\hline & 6649 & Ostrinia nubilalis & 1 & & 1 & \\
\hline & 6643 & Psammotis pulveralis & 1 & & & \\
\hline & 6655 & Anania verbascalis & & 1 & & \\
\hline & 6631 & Anania coronata & & & 1 & \\
\hline & 6658 & Anania hortulata & 1 & & & 1 \\
\hline & 6667 & Patania ruralis & 1 & & & \\
\hline & 6680 & Agrotera nemoralis & & & & 1 \\
\hline & 6682 & Diasemia reticularis & 1 & & 1 & \\
\hline & 6719 & Nomophila noctuella & 1 & & & \\
\hline
\end{tabular}




\begin{tabular}{|c|c|c|c|c|c|c|}
\hline \multirow[t]{2}{*}{ Suprafamily } & \multirow{2}{*}{$\begin{array}{c}\text { K \& R } \\
\text { Number }\end{array}$} & \multirow[t]{2}{*}{ Taxon } & \multicolumn{4}{|c|}{ County } \\
\hline & & & Ilfov & Dâmbovița & Giurgiu & Argeș \\
\hline \multirow{6}{*}{ Drepanoidea } & 7483 & Habrosyne pyritoides & 1 & & & \\
\hline & 7486 & Tethea or & 1 & 1 & & \\
\hline & 7494 & Polyploca ridens & 1 & & & \\
\hline & 7496 & Asphalia ruficollis & 1 & & & \\
\hline & 7508 & Drepana falcataria & & 1 & & \\
\hline & 7512 & Cilix glaucata & 1 & & & \\
\hline \multirow{4}{*}{ Lasiocampoidea } & 6743 & Malacosoma neustria & 1 & & & \\
\hline & 6752 & Lasiocampa quercus & 1 & & & \\
\hline & 6777 & Gastropacha quercifolia & 1 & & & \\
\hline & 6780 & Odonestis pruni & 1 & & & \\
\hline \multirow{9}{*}{ Bombycoidea } & 6788 & Aglia tau & 1 & & & \\
\hline & 6793 & Saturnia pyri & 1 & 1 & & \\
\hline & 6794 & Saturnia pavonia & 1 & & & \\
\hline & 6822 & Smerinthus ocellata & 1 & & & \\
\hline & 6824 & Laothoe populi & 1 & & & \\
\hline & 6828 & Agrius convolvuli & 1 & & & \\
\hline & 6843 & Macroglossum stellatarum & 1 & & & \\
\hline & 6853 & Hyles euphorbiae & 1 & & & \\
\hline & 6862 & Deilephila elpenor & 1 & & & \\
\hline \multirow{34}{*}{ Geometroidea } & 7522 & Abraxas grossulariata & 1 & & 1 & \\
\hline & 7530 & Ligdia adustata & 1 & & 1 & \\
\hline & 7527 & Lomaspilis marginata & 1 & 1 & 1 & \\
\hline & 7534 & Stegania dilectaria & 1 & & 1 & \\
\hline & 7539 & Macaria notata & 1 & & & \\
\hline & 7540 & Macaria alternata & 1 & & 1 & \\
\hline & 7547 & Chiasmia clathrata & & 1 & 1 & \\
\hline & 7607 & Plagodis dolabraria & 1 & & 1 & \\
\hline & 7618 & Therapis flavicaria & 1 & 1 & & \\
\hline & 7620 & Pseudopanthera macularia & & 1 & & \\
\hline & 7613 & Opisthograptis luteolata & & 1 & & \\
\hline & 7633 & Ennomos quercinaria & & & & 1 \\
\hline & 7635 & Ennomos fuscantaria & 1 & & 1 & \\
\hline & 7636 & Ennomos erosaria & 1 & & & \\
\hline & 7641 & Selenia dentaria & & 1 & & \\
\hline & 7642 & Selenia lunularia & 1 & & 1 & \\
\hline & 7652 & Crocallis tusciaria & 1 & & & \\
\hline & 7659 & Ourapteryx sambucaria & 1 & & & \\
\hline & 7663 & Colotois pennaria & 1 & 1 & 1 & \\
\hline & 7824 & Cabera pusaria & & & 1 & \\
\hline & 7826 & Cabera exanthemata & 1 & & & \\
\hline & 7828 & Lomographa bimaculata & 1 & & & \\
\hline & 7829 & Lomographa temerata & 1 & & & \\
\hline & 7665 & Angerona prunaria & 1 & & & \\
\hline & 7953 & Alsophila aescularia & 1 & & & \\
\hline & 7954 & Alsophila aceraria & 1 & 1 & & \\
\hline & 7672 & Phigalia pilosaria & 1 & & & \\
\hline & 7674 & Lycia hirtaria & 1 & & & \\
\hline & 7681 & Lycia pomonaria & 1 & & & \\
\hline & 7685 & Biston strataria & 1 & & & \\
\hline & 7686 & Biston betularia & 1 & & & \\
\hline & 7693 & Agriopis leucophaearia & 1 & & & \\
\hline & 7695 & Agriopis aurantiaria & 1 & 1 & & \\
\hline & 7696 & Agriopis marginaria & 1 & & & \\
\hline
\end{tabular}




\begin{tabular}{|c|c|c|c|c|c|c|}
\hline \multirow[t]{2}{*}{ Suprafamily } & \multirow{2}{*}{$\begin{array}{c}\text { K\& R } \\
\text { Number }\end{array}$} & \multirow[t]{2}{*}{ Taxon } & \multicolumn{4}{|c|}{ County } \\
\hline & & & Ilfov & Dâmbovița & Giurgiu & Argeș \\
\hline & 7699 & Erannis defoliaria & 1 & 1 & & \\
\hline & 7777 & Alcis repandata & 1 & & & \\
\hline & 7783 & Hypomecis roboraria & 1 & & & \\
\hline & 7784 & Hypomecis punctinalis & 1 & & & \\
\hline & 7794 & Ascotis selenaria & 1 & 1 & & \\
\hline & 7796 & Ectropis crepuscularia & 1 & 1 & & \\
\hline & 7802 & Aethalura punctulata & & 1 & & \\
\hline & 7804 & Ematurga atomaria & 1 & & & 1 \\
\hline & 7965 & Pseudoterpna pruinata & & 1 & & \\
\hline & 7971 & Comibaena bajularia & 1 & & & \\
\hline & 7975 & Thetidia smaragdaria & 1 & & & \\
\hline & 7980 & Hemithea aestivaria & & 1 & & \\
\hline & 7982 & Chlorissa viridata & 1 & & & \\
\hline & 7998 & Thalera fimbrialis & 1 & & & \\
\hline & 8000 & Hemistola chrysoprasaria & 1 & & & \\
\hline & 8002 & Jodis lactearia & 1 & & 1 & \\
\hline & 8100 & Idaea serpentata & & 1 & & 1 \\
\hline & 8104 & Idaea muricata & 1 & & & \\
\hline & 8107 & Idaea rusticata & 1 & & & \\
\hline & 8134 & Idaea inquinata & & 1 & & \\
\hline & 8142 & Idaea politaria & 1 & & & \\
\hline & 8184 & Idaea aversata & 1 & & 1 & \\
\hline & 8036 & Scopula immorata & & 1 & & \\
\hline & 8037 & Scopula tessellaria & 1 & & & \\
\hline & 8038 & Scopula corrivalaria & 1 & & & \\
\hline & 8042 & Scopula nigropunctata & 1 & & 1 & \\
\hline \multirow{27}{*}{ Geometroidea } & 8054 & Scopula rubiginata & & & 1 & \\
\hline & 8059 & Scopula marginepunctata & 1 & & & \\
\hline & 8064 & Scopula immutata & 1 & 1 & 1 & \\
\hline & 8068 & Scopula flaccidaria & 1 & & & \\
\hline & 8028 & Timandra comae & 1 & & & \\
\hline & 8012 & Cyclophora pendularia & 1 & & & \\
\hline & 8013 & Cyclophora albiocellaria & 1 & & 1 & \\
\hline & 8014 & Cyclophora annularia & 1 & & 1 & \\
\hline & 8018 & Cyclophora ruficiliaria & 1 & & & \\
\hline & 8019 & Cyclophora porata & 1 & & & \\
\hline & 8022 & Cyclophora punctaria & 1 & 1 & 1 & \\
\hline & 8024 & Cyclophora linearia & & & 1 & \\
\hline & 8221 & Lythria purpuraria & 1 & 1 & 1 & \\
\hline & 8240 & Scotopteryx mucronata & & & & 1 \\
\hline & 8256 & Xanthorhoe fluctuata & 1 & & & \\
\hline & 8253 & Xanthorhoe ferrugata & 1 & 1 & & \\
\hline & 8289 & Camptogramma bilineata & & 1 & & \\
\hline & 8275 & Epirrhoe alternata & 1 & 1 & & \\
\hline & 8277 & Epirrhoe rivata & & & 1 & \\
\hline & 8350 & Cidaria fulvata & & 1 & & \\
\hline & 8335 & Gandaritis pyraliata & & 1 & & \\
\hline & 8385 & Colostygia pectinataria & 1 & & & \\
\hline & 8447 & Operophtera brumata & 1 & 1 & & \\
\hline & 8663 & Minoa murinata & & & 1 & \\
\hline & 8656 & Asthena albulata & & & 1 & \\
\hline & 8658 & Asthena anseraria & & 1 & 1 & \\
\hline & 8660 & Hydrelia flammeolaria & & 1 & 1 & \\
\hline
\end{tabular}




\begin{tabular}{|c|c|c|c|c|c|c|}
\hline \multirow[t]{2}{*}{ Suprafamily } & \multirow{2}{*}{$\begin{array}{c}\text { K \& R } \\
\text { Number }\end{array}$} & \multirow[t]{2}{*}{ Taxon } & \multicolumn{4}{|c|}{ County } \\
\hline & & & Ilfov & Dâmbovița & Giurgiu & Argeș \\
\hline \multirow{9}{*}{ Geometroidea } & 8432 & Philereme vetulata & & 1 & 1 & \\
\hline & 8401 & Horisme corticata & & 1 & & \\
\hline & 8411 & Melanthia procellata & & 1 & & \\
\hline & 8639 & Lithostege farinata & 1 & & & \\
\hline & 8665 & Lobophora halterata & & 1 & & \\
\hline & 8599 & Gymnoscelis rufifasciata & 1 & & & \\
\hline & 8601 & Chloroclystis v-ata & 1 & & 1 & \\
\hline & 8479 & Eupithecia plumbeolata & 1 & & & \\
\hline & 8536 & Eupithecia denotata & & 1 & & \\
\hline \multirow{44}{*}{ Noctuoidea } & 8698 & Clostera curtula & 1 & 1 & & \\
\hline & 8699 & Clostera pigra & 1 & & & \\
\hline & 8700 & Clostera anachoreta & 1 & & & \\
\hline & 8716 & Notodonta dromedarius & & & & 1 \\
\hline & 8718 & Notodonta tritophus & & 1 & & \\
\hline & 8719 & Notodonta ziczac & & 1 & & \\
\hline & 8722 & Drymonia ruficornis & 1 & & & \\
\hline & 8727 & Pheosia tremula & 1 & 1 & & \\
\hline & 8732 & Pterostoma palpina & 1 & & & \\
\hline & 8738 & Ptilodon capucina & 1 & & & \\
\hline & 8739 & Ptilodon cucullina & & 1 & & \\
\hline & 8708 & Furcula furcula & 1 & 1 & & \\
\hline & 8710 & Furcula bifida & 1 & & & \\
\hline & 8750 & Phalera bucephala & 1 & & & \\
\hline & 8762 & Spatalia argentina & 1 & & & \\
\hline & 9008 & Rivula sericealis & & 1 & & \\
\hline & 9147 & Eublemma purpurina & 1 & & 1 & \\
\hline & 8839 & Paracolax tristalis & 1 & & 1 & \\
\hline & 8846 & Herminia grisealis & 1 & & & \\
\hline & 8858 & Herminia tarsipennalis & 1 & & & \\
\hline & 8849 & Polypogon tentacularia & & 1 & & 1 \\
\hline & 8856 & Zanclognatha lunalis & 1 & & 1 & \\
\hline & 8857 & Zanclognatha zelleralis & 1 & & & \\
\hline & 8852 & Pechypogo strigilata & 1 & & & \\
\hline & 8994 & Hypena proboscidalis & 1 & & & \\
\hline & 8995 & Hypena rostralis & 1 & & & \\
\hline & 9006 & Phytometra viridaria & & & & 1 \\
\hline & 9018 & Colobochyla salicalis & 1 & & 1 & \\
\hline & 9169 & Trisateles emortualis & 1 & & & \\
\hline & 8984 & Scoliopteryx libatrix & 1 & & & \\
\hline & 8877 & Catocala elocata & & 1 & & \\
\hline & 8897 & Minucia lunaris & 1 & & & \\
\hline & 8909 & Grammodes stolida & 1 & & & \\
\hline & 8969 & Euclidia glyphica & 1 & & 1 & \\
\hline & 10479 & Pelosia muscerda & 1 & & & \\
\hline & 10480 & Pelosia obtusa & 1 & & & \\
\hline & 10494 & Eilema palliatella & 1 & & 1 & \\
\hline & 10517 & Amata phegea & & & 1 & \\
\hline & 10521 & Dysauxes ancilla & 1 & & & \\
\hline & 10550 & Phragmatobia fuliginosa & 1 & & & \\
\hline & 10566 & Spilarctia lutea & 1 & & & \\
\hline & 10567 & Spilosoma lubricipeda & 1 & & & \\
\hline & 10570 & Hyphantria cunea & 1 & & & \\
\hline & 10579 & Diacrisia purpurata & 1 & & & \\
\hline
\end{tabular}




\begin{tabular}{|c|c|c|c|c|c|c|}
\hline \multirow[t]{2}{*}{ Suprafamily } & \multirow{2}{*}{$\begin{array}{c}\text { K \& R } \\
\text { Number }\end{array}$} & \multirow[t]{2}{*}{ Taxon } & \multicolumn{4}{|c|}{ County } \\
\hline & & & Ilfov & Dâmbovița & Giurgiu & Argeș \\
\hline & 10583 & Diacrisia sannio & & & & 1 \\
\hline & 10600 & Arctia villica & 1 & & & \\
\hline & 10414 & Leucoma salicis & 1 & & & \\
\hline & 10387 & Calliteara pudibunda & & & 1 & \\
\hline & 10397 & Orgyia antiqua & 1 & & 1 & \\
\hline & 10408 & Penthophera morio & & 1 & & \\
\hline & 10372 & Clocasia coryli & 1 & & & \\
\hline & 8793 & Simyra albovenosa & 1 & & & \\
\hline & 8778 & Acronicta aceris & 1 & & & \\
\hline & 8780 & Acronicta megacephala & 1 & & & \\
\hline & 8783 & Acronicta auricoma & 1 & & & \\
\hline & 8787 & Acronicta rumicis & 1 & & & \\
\hline & 9091 & Abrostola tripartita & 1 & & & \\
\hline & 9093 & Abrostola triplasia & 1 & & & \\
\hline & 9051 & Macdunnoughia confusa & 1 & & & \\
\hline & 9046 & Diachrysia stenochrysis & 1 & & & \\
\hline & 9055 & Autographa gamma & 1 & & & \\
\hline & 9053 & Plusia festucae & 1 & & & \\
\hline & 9114 & Deltote pygarga & 1 & 1 & 1 & \\
\hline & 9118 & Deltote bankiana & 1 & & & \\
\hline & 10423 & Meganola strigula & 1 & & & \\
\hline & 10451 & Pseudoips prasinana & 1 & & 1 & \\
\hline & 9338 & Panemeria tenebrata & 1 & & & \\
\hline & 8965 & Tyta luctuosa & 1 & & 1 & \\
\hline & 9097 & Acontia trabealis & 1 & 1 & & \\
\hline & 8958 & Aedia funesta & 1 & & & \\
\hline \multirow[t]{27}{*}{ Noctuoidea } & 9199 & Cucullia umbratica & 1 & 1 & & \\
\hline & 9240 & Calophasia lunula & 1 & & & \\
\hline & 9311 & Amphipyra tragopoginis & 1 & & & \\
\hline & 9320 & Asteroscopus sphinx & 1 & & 1 & \\
\hline & 9682 & Allophyes oxyacanthae & 1 & & & \\
\hline & 9358 & Protoschinia scutosa & 1 & & 1 & \\
\hline & 9364 & Heliothis viriplaca & 1 & & & \\
\hline & 9122 & Pseudeustrotia candidula & 1 & & 1 & \\
\hline & 9417 & Caradrina morpheus & 1 & & & \\
\hline & 9433 & Caradrina clavipalpis & 1 & & & \\
\hline & 9450 & Hoplodrina blanda & 1 & & & \\
\hline & 9453 & Hoplodrina respersa & 1 & & & \\
\hline & 9454 & Hoplodrina ambigua & 1 & & & \\
\hline & 9455 & Charanyca trigrammica & 1 & & & \\
\hline & 9475 & Athetis furvula & 1 & & & \\
\hline & 9505 & Phlogophora meticulosa & 1 & & & \\
\hline & 9557 & Tiliacea aurago & 1 & & & \\
\hline & 9559 & Cirrhia icteritia & 1 & & & \\
\hline & 9560 & Cirrhia gilvago & 1 & & & \\
\hline & 9561 & Cirrhia ocellaris & 1 & & & \\
\hline & 9566 & Sunira circellaris & 1 & & & \\
\hline & 9569 & Agrochola lota & 1 & & & \\
\hline & 9571 & Agrochola macilenta & 1 & & & \\
\hline & 9601 & Conistra ligula & & & & 1 \\
\hline & 9611 & Conistra erythrocephala & 1 & & & \\
\hline & 9596 & Eupsilia transversa & 1 & & & \\
\hline & 9841 & Gortyna flavago & 1 & & & \\
\hline
\end{tabular}




\begin{tabular}{|c|c|c|c|c|c|c|}
\hline \multirow[t]{2}{*}{ Suprafamily } & \multirow{2}{*}{$\begin{array}{c}\text { K \& R } \\
\text { Number }\end{array}$} & \multirow[t]{2}{*}{ Taxon } & \multicolumn{4}{|c|}{ County } \\
\hline & & & Ilfov & Dâmbovița & Giurgiu & Argeș \\
\hline \multirow{37}{*}{ Noctuoidea } & 9834 & Hydraecia micacea & 1 & & & \\
\hline & 9814 & Rhizedra lutosa & 1 & & & \\
\hline & 9870 & Sedina buettneri & 1 & & & \\
\hline & 9859 & Nonagria typhae & 1 & & & \\
\hline & 9771 & Apamea sordens & 1 & & & \\
\hline & 9781 & Oligia versicolor & 1 & & & \\
\hline & 9782 & Oligia latruncula & 1 & & & \\
\hline & 9895 & Anarta trifolii & 1 & & & \\
\hline & 9920 & Lacanobia suasa & 1 & & & \\
\hline & 9917 & Lacanobia oleracea & 1 & & & \\
\hline & 9987 & Mamestra brassicae & 1 & & & \\
\hline & 9927 & Hecatera dysodea & 1 & & & \\
\hline & 9933.1 & Hadena capsincola & 1 & & & \\
\hline & 9957 & Hadena perplexa & 1 & & & \\
\hline & 10065 & Tholera decimalis & 1 & & & 1 \\
\hline & 9999 & Mythimna turca & 1 & & & \\
\hline & 10004 & Mythimna pudorina & 1 & & & \\
\hline & 10007 & Mythimna pallens & 1 & & & \\
\hline & 10003 & Mythimna vitellina & 1 & & & \\
\hline & 10002 & Mythimna albipuncta & 1 & & & \\
\hline & 10019 & Mythimna congrua & 1 & & & \\
\hline & 10010 & Leucania obsoleta & 1 & & & \\
\hline & 10017 & Senta flammea & 1 & & & \\
\hline & 10037 & Orthosia incerta & 1 & & & \\
\hline & 10039 & Orthosia cruda & 1 & & & \\
\hline & 10044 & Orthosia cerasi & 1 & 1 & & \\
\hline & 10048 & Orthosia gracilis & 1 & & & \\
\hline & 10038 & Orthosia gothica & 1 & 1 & & \\
\hline & 10351 & Agrotis segetum & 1 & & & \\
\hline & 10086 & Ochropleura plecta & 1 & & & \\
\hline & 10139 & Rhyacia simulans & 1 & & & \\
\hline & 10096 & Noctua pronuba & 1 & & & \\
\hline & 10098 & Noctua interposita & 1 & & & \\
\hline & 10163 & Spaelotis ravida & 1 & & & \\
\hline & 10199 & Xestia c-nigrum & 1 & & & \\
\hline & 10212 & Xestia xanthographa & 1 & & & \\
\hline & 10224 & Cerastis rubricosa & 1 & & & \\
\hline
\end{tabular}

